\title{
Avaliação da qualidade da casca dos ovos provenientes de matrizes pesadas com diferentes idades
}

\author{
[Evaluation of eggshell quality from broiler breeder hens with different ages] \\ V.M. Barbosa ${ }^{1 *}$, N.C. Baião ${ }^{2}$, P.M.M. Mendes ${ }^{3}$, J.S.R. Rocha ${ }^{3}$, M.A. Pompeu ${ }^{3}$, L.J.C. Lara ${ }^{2}$, \\ N.R.S. Martins ${ }^{2}$, D.L. Nelson ${ }^{4}$, D.J.A. Miranda ${ }^{3}$, C.E. Cunha ${ }^{3}$, \\ D.M. Cardoso $^{3}$, P.C. Cardeal
${ }^{1}$ Aluna de pós-doutorado - Instituto Nacional de Ciência e Tecnologia - Informação Genético-Sanitária da Pecuária Brasileira/ INCT - Pecuária - Escola de Veterinária - UFMG - Bolsista CNPq
${ }^{2}$ Escola de Veterinária - Universidade Federal de Minas Gerais - Belo Horizonte, MG
${ }^{3}$ Aluno de pós-graduação - Escola de Veterinária - Universidade Federal de Minas Gerais - Belo Horizonte, MG
${ }^{4}$ Farmácia - Universidade Federal de Minas Gerais - Belo Horizonte, MG

\begin{abstract}
RESUMO
Foi realizado um estudo sobre qualidade da casca dos ovos incubáveis provenientes de matrizes pesadas com diferentes idades, por meio da avaliação da relação entre peso da casca e peso do ovo e análises de peso específico, espessura, porosidade, resistência e microscopia eletrônica. Os dois tratamentos foram definidos pela idade da matriz, sendo considerados ovos de matrizes novas - 33 semanas - e de matrizes velhas - 63 semanas. Os ovos de matrizes com 33 semanas foram mais leves, e o número de poros por $\mathrm{cm}^{2}$ foi menor que o de ovos das aves mais velhas. Ovos de aves com 63 semanas apresentaram menor percentual de casca em relação ao peso do ovo, menor peso específico e menores resistência e espessura da casca. A proporção das membranas da casca em relação à sua espessura total foi maior nas matrizes mais novas. Concluiu-se que ovos de matrizes velhas têm qualidade de casca inferior aos ovos das matrizes novas e que as membranas da casca nos ovos de matrizes novas desempenham papel relevante em sua estrutura.
\end{abstract}

Palavras-chave: incubação, matrizes pesadas, qualidade de casca do ovo

\begin{abstract}
We conducted a study on eggshell quality from $C o b b{ }^{\circledR}$ broiler breeders at different ages by assessing the relationship between eggshell weight and egg weight and analysis of specific weight, thickness, porosity, strength and electron microscopy. Both treatments were defined by broiler breeder age, considering eggs from young breeders (33 weeks) and eggs from old breeders (63 weeks). It was observed that eggs from breeders at 33 weeks have lower weight and number of pores per $\mathrm{cm}^{2}$ compared with eggs from older birds. 63 weeks broiler breeders had lower percentage of eggshell in relation to egg weight, lower specific weight, strength and thickness, when compared with eggs from young breeders. The proportion of shell membranes in relation to its total thickness was significantly higher in young breeders. It was concluded that eggs from older broiler breeders have lower eggshell quality than the young breeders. The shell membranes in young breeder's eggs play a significant role in its structure, making it necessary to focus on this layer in future studies on eggshell quality and their relationship between breeder age and incubation yield.
\end{abstract}

Keywords: broiler breeders, eggshell quality, incubation

Recebido em 4 de dezembro de 2011

Aceito em 8 de fevereiro de 2012

E-mail: vmichalsky@gmail.com 


\section{INTRODUÇÃ̃O}

A casca do ovo é um dos fatores que exercem papel fundamental no processo de incubação, oferecendo proteção contra a invasão microbiana, controlando a troca de água e gases através dos poros e constituindo a fonte de cálcio para o embrião durante seu desenvolvimento. $\mathrm{O}$ êxito destas funções é dependente de sua adequada formação e de sua composição estrutural (Romanoff e Romanoff, 1949; Rahn et al., 1981).

A estrutura da casca é perfeitamente ordenada, dividida em camadas, e resulta de uma deposição sequencial de fração orgânica, 3,5\%, e mineral, $96,5 \%$, que ocorre nos segmentos istmo e útero da galinha, durante um período predeterminado. A porção orgânica da casca consiste nas membranas da casca, nos sítios mamilares de nucleação e na cutícula. A fração calcificada é composta pela camada mamilar (ou camada de botões mamilares), camada em paliçada e camada de cristal vertical (Parsons, 1982). Os poros são cavidades em forma de funis, amplos na superfície da casca e que se estreitam, formando canais que penetram as camadas de cristal e terminam em fissuras adjacentes aos botões mamilares (Tullett e Deeming,1982; Nys et al., 1999; Hunton, 2005).

De acordo com McDaniel et al. (1979) e Hamilton (1982), o tamanho e o peso do ovo aumentam com a idade das aves, mas o peso da casca não aumenta na mesma proporção. Em consequência, à medida que a ave envelhece, a estrutura da casca sofre modificações que demonstram queda na qualidade dela. McLoughlin e Gous (2000) ratificaram a necessidade de compreensão destas alterações na estrutura da casca do ovo de acordo com a idade da matriz, para aplicá-las efetivamente no manejo da incubação.

Os critérios comumente utilizados para se avaliar a qualidade da casca baseiam-se nas medições do peso específico, espessura, resistência e relação entre o peso da casca e o peso do ovo (Orband e Roland Sr., 1990). Segundo Emara (2008), o uso de microscopia eletrônica tem fornecido informações relevantes sobre a composição da casca, e os resultados destas análises ultraestruturais evidenciam o conceito de que as propriedades mecânicas da casca não deveriam ser definidas apenas pelas análises citadas anteriormente. $\mathrm{O}$ autor destacou que a microscopia eletrônica pode auxiliar na compreensão dos efeitos da qualidade da casca e em suas relações entre idade da matriz e rendimento de incubação.

O objetivo deste trabalho foi realizar um estudo sobre a qualidade da casca dos ovos incubáveis provenientes de matrizes pesadas novas e velhas, por meio da avaliação da relação entre o peso da casca e o peso do ovo e análises de peso específico, espessura, porosidade, resistência e microscopia eletrônica da casca.

\section{MATERIAL E MÉTODOS}

Os ovos foram obtidos de dois lotes de matrizes pesadas da linhagem $\mathrm{Cobb} \AA$, com idades de $33 \mathrm{e}$ 63 semanas, produzidos no mesmo dia e provenientes da segunda e terceira coletas na granja. Para cada idade de matriz foi retirada e identificada uma amostra de 50 ovos para as avaliações de peso específico e porcentagem de casca; uma amostra de 50 ovos para as avaliações de resistência, espessura e porosidade da casca; e uma amostra de 10 ovos para as avaliações ultraestruturais da casca.

Os tratamentos foram definidos pela idade da matriz, sendo: $M N$ : ovos incubáveis de matrizes novas - 33 semanas - e $M V$ : ovos incubáveis de matrizes velhas -63 semanas.

As avaliações de peso específico foram realizadas um dia após a postura. O método para a análise foi baseado no princípio de Arquimedes, em que os valores de peso específico, expressos em $\mathrm{g} / \mathrm{mL} \mathrm{H}_{2} \mathrm{O}$, foram obtidos pela fórmula:

\section{(Peso do ovo no ar) \\ (Peso do ovo no ar - Peso do ovo em água)}

O equipamento utilizado para esta avaliação foi montado com uma balança de precisão de $0,5 \mathrm{~g}$ e suporte de ferro usado para sustentar um recipiente contendo água destilada, que possuía espaço adequado para a pesagem do ovo no ar. Lateralmente, foi colocada outra estrutura de ferro, da qual descia uma haste com aro apropriado para a pesagem do ovo dentro da água. $\mathrm{O}$ equipamento foi colocado sobre a 
balança, que, em seguida, foi zerada. Iniciou-se, então, a pesagem dos ovos, sempre com a balança zerada antes da próxima pesagem. Os pesos dos ovos dentro e fora da água foram anotados para o cálculo posterior do peso específico.

Para as avaliações das proporções de casca, os mesmos ovos utilizados para as avaliações do peso específico foram pesados em balança com precisão de $0,01 \mathrm{~g}$ e posteriormente quebrados, sendo seus componentes separados em copos plásticos e identificados. As cascas, depois de lavadas em água corrente para retirada de resíduos do albúmen, secaram em temperatura ambiente durante 24 horas e foram pesadas individualmente. Os percentuais de casca foram calculados dividindo-se os valores obtidos pelo peso do ovo e multiplicando-se por 100.

Nas avaliações de resistência da casca, utilizouse uma nova amostra de 50 ovos para cada idade de matriz. A força necessária para quebrar a casca, em gramas, foi determinada por meio do aparelho TA.X T2 Texture Analyser (Stable Micro Systems, Surrey, England), pertencente ao Setor de Análise de Alimentos, da Faculdade de Farmácia da UFMG. Foi utilizada uma sonda P4 DIA Cylinder de aço inoxidável de $4 \mathrm{~mm}$ de diâmetro, com distância de $6 \mathrm{~mm}$ e velocidade pré, durante e pós-teste de 3,$0 ; 0,5$; e $5,0 \mathrm{~mm} / \mathrm{s}$, respectivamente. A força de gatilho da sonda foi de $3,0 \mathrm{~g}$. O teste seguiu o método de fratura por compressão, em que o ovo inteiro é colocado longitudinalmente, segundo Rodriguez-Navarro (2002), sobre um suporte de metal em forma de anel com $5 \mathrm{~cm}$ de diâmetro dentro de um cadinho de porcelana. A casca é pressionada até que ocorra a fratura, e a força necessária usada é a indicadora da resistência da casca.

A medida da espessura da casca foi realizada com um micrômetro digital (Mitutoyo, resolução $0,001 \mathrm{~mm})$. O aparelho foi cedido pelo Departamento de Mecânica da Faculdade de Engenharia da UFMG. A mensuração foi efetuada nos 50 ovos de cada idade de matriz utilizados para a análise de resistência. Estas cascas foram separadas com auxílio de uma tesoura em três partes, correspondentes às regiões apical - extremidade afilada -, equatorial e basal - extremidade alargada que contém a câmara de ar. Foi medida a espessura de cada região, e a espessura média da casca do ovo foi calculada por meio da média das três regiões.

Os ovos utilizados para as medições de resistência e espessura da casca também foram usados para a determinação do número de poros, pelo método de Rahn et al. (1981). Cada uma das regiões apical, equatorial e basal foram colocadas separadamente em béquer e fervidas por 10 minutos em solução aquosa de $\mathrm{NaOH} 5 \%$ para remoção da cutícula e das membranas da casca. Em seguida, foram mergulhadas rapidamente em um recipiente contendo água e secas à temperatura ambiente durante duas horas. Após a secagem, as amostras foram coradas por meio de conta-gotas com solução aquosa de azul de metileno (1\%) por dois minutos, lavadas rapidamente em água corrente e secas à temperatura ambiente por mais uma hora. Em cada fragmento foi demarcada com um molde uma área de $1 \mathrm{~cm}^{2}$, dividida em quatro áreas de $25 \mathrm{~mm}^{2}$. O número de poros foi contado sob estereomicroscópio, sendo a soma das quatro áreas de $25 \mathrm{~mm}^{2}$ considerada o número de poros por $\mathrm{cm}^{2}$ para cada uma das regiões apical, equatorial e basal. A quantidade de poros por $\mathrm{cm}^{2}$ em cada ovo foi calculada por meio da média das três regiões.

Para as avaliações ultraestruturais das camadas da casca do ovo, foram selecionados 10 ovos que tinham os pesos próximos ao peso médio obtido para cada idade da matriz. Estes ovos foram levemente quebrados, e as cascas foram segmentadas, com auxílio de uma tesoura, em três partes, correspondentes às regiões apical, equatorial e basal. O albúmen residual foi removido mergulhando-se rapidamente os segmentos em béquer contendo água e, posteriormente, as cascas foram secas em temperatura ambiente. Em seguida, fragmentos de aproximadamente $0,5 \mathrm{~cm}^{2}$ foram retirados de cada uma das regiões do ovo, armazenados em tubos ependorff e mantidos em geladeira por sete dias. Após este período, as amostras foram montadas horizontalmente em stubs de alumínio usando-se cola adesiva, sendo a região da cutícula da casca posicionada na região superior do stub. Estas amostras receberam, então, uma metalização de ouro de $35 \mathrm{~nm}$ por três minutos, por meio do aparelho Ion Scancoat 6 Sputter Coater® (Edwards, UK) e posteriormente foram examinadas no microscópio eletrônico de varredura Zeiss® DSM 950 (West Germany) 
com uma voltagem de aceleração de $20 \mathrm{~kW}$. A metalização foi realizada no Departamento de Microscopia e Microanálise do Instituto de Física da UFMG, e a montagem dos stubs assim como as avaliações de microscopia eletrônica foram realizadas no Centro de Microscopia Eletrônica do Instituto de Ciências Biológicas da UFMG. As camadas da casca do ovo (mamilar, paliçada e membranas) foram identificadas num aumento de 200x, a camada de cristal vertical num aumento de 2000x e a cutícula num aumento de 5000x (Fig. 1). Somente foram considerados os seis ovos que, no momento da análise, mantinham os fragmentos íntegros das regiões apical, equatorial e basal e que possibilitavam a visualização de todas as camadas. Para cada região do ovo, o comprimento de cada camada foi mensurado em três pontos e a média dos valores foi registrada. $\mathrm{O}$ valor médio da espessura das camadas e sua proporção em relação à espessura total da casca foram calculados pela média das regiões apical, equatorial e basal de cada ovo.

Para as análises de peso específico, percentual de casca em relação ao peso do ovo, resistência, espessura e porosidade da casca, foi utilizado o delineamento experimental inteiramente ao acaso, constituído por dois tratamentos (idade das matrizes) e 50 repetições por tratamento, sendo cada ovo considerado a repetição. Para as avaliações da espessura e porosidade da casca do ovo nas diferentes regiões, foi utilizado o delineamento inteiramente ao acaso, em esquema fatorial $2 \times 3$, sendo duas idades de matrizes e três regiões da casca, com 50 repetições por tratamento. Nas avaliações ultraestruturais das camadas da casca do ovo, o delineamento utilizado foi inteiramente ao acaso, constituído por dois tratamentos (idade das matrizes) e seis repetições por tratamento, sendo o ovo considerado a repetição. A normalidade e a homocedasticidade dos dados foram verificadas pelo teste de Lilliefors e Bartlett. Os dados normais e homogêneos foram submetidos às análises de variância, e as médias comparadas pelo teste F (Sampaio, 2002). As medianas das respostas não normais e não homogêneas foram comparadas pelos testes Mann-Whitney e
Kruskal-Wallis, utilizando-se o programa SAEG, versão 9.1 (Sistema..., 2005).

A metodologia utilizada neste experimento foi aprovada pelo Comitê de Ética em Experimentação Animal da Universidade Federal de Minas Gerais, sob protocolo ${ }^{\circ}$ 247/10.

\section{RESULTADOS E DISCUSSÃO}

Os resultados mostrados na Tab. 1 revelaram que os ovos das matrizes com idade de 63 semanas apresentaram menor peso específico $(\mathrm{P}<0,05)$ quando comparados aos ovos das matrizes de 33 semanas. Como a análise de peso específico é um indicador de qualidade do ovo, estes resultados demonstram uma melhor qualidade de casca nos ovos de matrizes mais novas, concordando com os resultados obtidos em trabalhos anteriores (McDaniel et al., 1979; Barbosa, 2005), com exceção de Luquetti et al. (2004), que não encontraram diferenças de peso específico nos ovos provenientes de matrizes pesadas de 30, 45 e 60 semanas de idade.

As matrizes mais velhas apresentaram menores percentuais de casca em relação ao peso do ovo $(\mathrm{P} \leq 0,005)$ do que as matrizes mais novas. Estes resultados estão de acordo com Rocha et al. (2008). Segundo Hamilton (1982), à medida que a ave envelhece, o peso da casca não aumenta na mesma proporção que o peso dos ovos. Brake (1996) explicou que esse fato pode ser atribuído à menor deposição de carbonato de cálcio por unidade de área, sendo que independentemente da progressão da idade, a quantidade de cálcio mobilizada para a formação da casca é a mesma.

Menor peso do ovo e do número de poros (Tab. 2) foi encontrado nos ovos de aves mais jovens $(\mathrm{P} \leq 0,001)$. Nestes, a espessura e a resistência das cascas foram maiores $(\mathrm{P} \leq 0,001)$ se comparadas às dos ovos das aves mais velhas. Verifica-se que, à medida que a ave aumentou de idade, as características físicas dos ovos revelaram mudanças estruturais que tornaram a qualidade da casca inferior. 


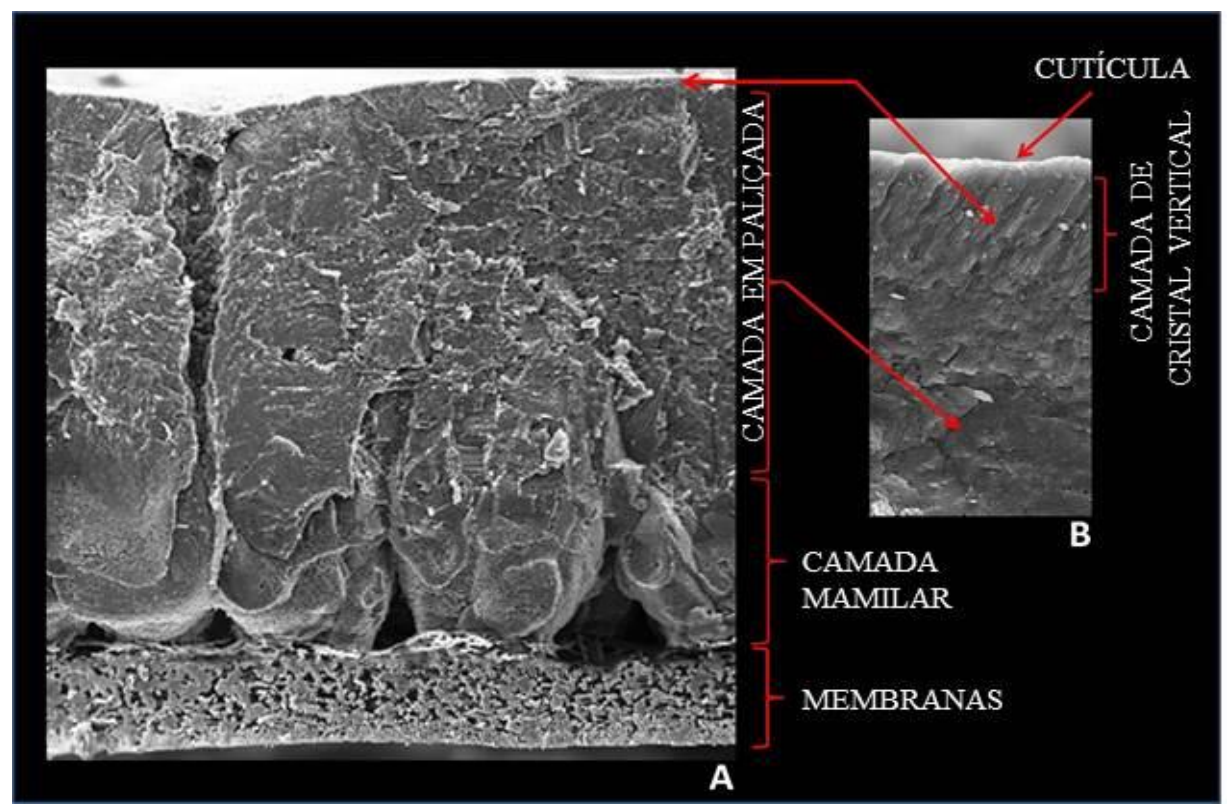

Figura 1. Fotomicrografia das camadas da casca do ovo por meio de microscopia eletrônica de varredura. Camadas mamilar, paliçada e membranas da casca em aumento de 200x (A). Camada de cristal vertical e cutícula em aumento de 2000x (B).

Tabela 1. Peso específico e porcentagem de casca em relação ao peso do ovo, de acordo com a idade da matriz

\begin{tabular}{ccc}
\hline Idade da matriz (semanas) & Peso específico $\left(\mathrm{g} / \mathrm{mL} \mathrm{H}_{2} \mathrm{O}\right)$ & Casca $(\%)$ \\
\hline 33 & $1,083 \mathrm{a}$ & $9,5 \mathrm{a}$ \\
63 & $1,080 \mathrm{~b}$ & $8,6 \mathrm{~b}$ \\
C.V $(\%)$ & 0,5 & 4,6 \\
\hline
\end{tabular}

Médias seguidas de letras distintas na coluna são diferentes pelo teste $\mathrm{F}(\mathrm{P} \leq 0,05)$.

Tabela 2. Peso médio do ovo, espessura, resistência e porosidade da casca, de acordo com a idade da matriz

\begin{tabular}{ccccc}
\hline $\begin{array}{c}\text { Idade da matriz } \\
(\text { semanas })\end{array}$ & $\begin{array}{c}\text { Peso do ovo } \\
(\mathrm{g})^{1}\end{array}$ & $\begin{array}{c}\text { Espessura da } \\
\text { casca }(\mathrm{mm})^{1}\end{array}$ & $\begin{array}{c}\text { Resistência da } \\
\text { casca }(\mathrm{g})^{1}\end{array}$ & $\begin{array}{c}\text { Poros da casca por } \mathrm{cm}^{2} \\
\left(\mathrm{n}^{\circ}\right)^{2}\end{array}$ \\
\hline 33 & $62,0 \mathrm{~B}$ & $0,457 \mathrm{~A}$ & $3193 \mathrm{~A}$ & $103 \mathrm{~B}$ \\
63 & $72,4 \mathrm{~A}$ & $0,435 \mathrm{~B}$ & $2994 \mathrm{~B}$ & $126 \mathrm{~A}$ \\
$\mathrm{CV}(\%)$ & 4,9 & 7,9 & 17,0 & - \\
\hline
\end{tabular}

Médias seguidas de letras distintas na coluna são diferentes pelo testes $\mathrm{F}^{1}$ e Mann-Whitney ${ }^{2}$, respectivamente $(\mathrm{P} \leq 0,001)$.

A influência da idade da matriz sobre o peso do ovo foi igualmente relatada por diversos autores (Vieira e Moran Jr., 1998; Peebles et al., 2000; Rocha et al., 2008). Segundo McLoughlin e Gous (2000), ovos produzidos por matrizes mais velhas são maiores, e, consequentemente, sua casca é mais fina. Também foi observado que ovos de matrizes com idade mais avançada têm maior número de poros, semelhante aos resultados apresentados na Tab. 2. Pesquisadores deram ênfase à informação de que um maior número de poros pode causar maior perda de calor ou desidratação do embrião, e o inverso pode dificultar a troca de gases entre o embrião e o meio externo. Segundo Peebles e Brake (1985), todas essas características estão diretamente 
relacionadas ao desenvolvimento e à mortalidade embrionária. Emara (2008) obteve dados semelhantes aos deste experimento, revelando que matrizes velhas produzem ovos com cascas de menor resistência quando comparadas com os de reprodutoras mais novas e que este é um fator importante a ser considerado no manuseio dos ovos férteis para evitar quedas na produtividade. espessura da casca na região da câmara de ar (região basal) revelou ser menor $(\mathrm{P} \leq 0,05)$ nas matrizes velhas (Tab. 3) quando comparada à das matrizes novas. Nas demais regiões da casca, não houve diferença $(\mathrm{P}>0,05)$ na espessura entre as idades das matrizes. As regiões da casca do ovo não influenciaram $(\mathrm{P}>0,05)$ esta variável tanto em matrizes novas como em matrizes velhas.

Houve interação entre a idade da matriz e as regiões da casca do ovo para esta variável. A

Tabela 3. Espessura da casca $(\mathrm{mm})$ em função da região do ovo e da idade da matriz

\begin{tabular}{cccc}
\hline $\begin{array}{c}\text { Idade da matriz } \\
\text { (semanas) }\end{array}$ & \multicolumn{3}{c}{ Regiões da casca do ovo } \\
\cline { 2 - 4 } 33 & $0,462 \mathrm{Aa}$ & Equatorial & Basal \\
63 & $0,440 \mathrm{Aa}$ & $0,448 \mathrm{Aa}$ & $\mathrm{Aa}$ \\
& $0,429 \mathrm{Aa}$ & $0,437 \mathrm{Ba}$
\end{tabular}

Médias seguidas de letras distintas, minúsculas na linha e maiúsculas na coluna, são diferentes pelo teste KruskalWallis $(\mathrm{P} \leq 0,05)$.

Independentemente da idade da matriz, foi encontrada menor $(\mathrm{P} \leq 0,05)$ porosidade na região apical - ponta mais fina do ovo - quando comparada às regiões equatorial e basal, que foram semelhantes entre si (Tab. 4). Estes dados estão de acordo com os relatados por BaxterJones (1991), mas diferentes dos de Hunton (1995) e La Scala Jr. (2003), que afirmaram que o maior número de poros se encontra na região basal quando comparado ao número de outras regiões da casca. Tazawa e Whittow (2000) descreveram que, durante a incubação, o ar do meio externo provoca aumento da câmara de ar através dos poros da casca, ao mesmo tempo em que ocorre perda de umidade do ovo para o ambiente. Porém, Stanishevskaya (2006) verificou que ao $13^{\circ}$ e $19^{\circ}$ dias de incubação, ocorre maior atividade de penetração de $\mathrm{O}_{2}$ na região equatorial do ovo. Os dados da Tab. 4 sugerem a hipótese de que distribuição dos poros pode ocorrer em razão da necessidade funcional relatada nas pesquisas desses autores. Matrizes mais velhas apresentaram maior $(\mathrm{P} \leq 0,05)$ número de poros na casca do que matrizes novas, independentemente da região do ovo (Tab. 2).

De acordo com os resultados da Tab. 5, os dados de espessura total da casca e a região calcificada, compreendida pelas camadas vertical, paliçada e mamilar, em matrizes de 33 semanas, foram maiores $(\mathrm{P} \leq 0,01)$ quando estas matrizes foram comparadas às de 63 semanas, semelhantemente aos resultados obtidos por esta técnica nos estudos de Ruiz e Lunam (2000) e RodriguezNavarro et al. (2002). Esta análise, feita por microscopia eletrônica, confirmou a diferença significativa encontrada para esta variável por meio da medição pelo micrômetro de forma manual (Tab. 2). Em relação às camadas da casca, a camada de cristal vertical foi a única porção da casca em que os ovos das matrizes novas revelaram maior espessura $(\mathrm{P} \leq 0,01)$ que as matrizes velhas, porém esta diferença não foi suficiente para alterar os resultados descritos anteriormente.

Tabela 4. Porosidade da casca em função da região do ovo e da idade da matriz

\begin{tabular}{ccccc} 
Idade da matriz & \multicolumn{2}{c}{ Porosidade da casca do ovo $\left(\mathrm{n}^{\circ}\right.$ de poros $\left./ \mathrm{cm}^{2}\right)$} & \multirow{2}{*}{ Média } \\
\cline { 2 - 4 } (semanas) & Apical & Equatorial & Basal & $103 \mathrm{~B}$ \\
\hline 33 & 91 & 104 & 114 & $126 \mathrm{~A}$ \\
63 & 103 & 130 & 148 & \\
\hline Média & $97 \mathrm{~b}$ & $117 \mathrm{a}$ & $131 \mathrm{a}$ & \\
\hline
\end{tabular}

Médias seguidas de letras distintas, minúsculas na linha e maiúsculas na coluna, são diferentes pelo teste KruskalWallis $(\mathrm{P} \leq 0,05)$. 
Tabela 5. Avaliação ultraestrutural da espessura $(\mu \mathrm{m})$ da casca do ovo, de acordo com as regiões e com a idade da matriz

\begin{tabular}{cccccccc}
$\begin{array}{c}\text { Idade } \\
\text { (sem) }\end{array}$ & Cutícula & $\begin{array}{c}\text { Camada } \\
\text { vertical }\end{array}$ & $\begin{array}{c}\text { Camada } \\
\text { paliçada }\end{array}$ & $\begin{array}{c}\text { Camada } \\
\text { mamilar }\end{array}$ & $\begin{array}{c}\text { Região } \\
\text { calcificada }\end{array}$ & Membrana & $\begin{array}{c}\text { Total } \\
\text { casca }\end{array}$ \\
\hline 33 & $3,13 \mathrm{a}$ & $12,85 \mathrm{~b}$ & $264,61 \mathrm{a}$ & $121,14 \mathrm{a}$ & $398,60 \mathrm{a}$ & $80,72 \mathrm{a}$ & $479,3 \mathrm{a}$ \\
63 & $2,78 \mathrm{~b}$ & $18,16 \mathrm{a}$ & $243,97 \mathrm{~b}$ & $111,09 \mathrm{~b}$ & $373,22 \mathrm{~b}$ & $52,32 \mathrm{~b}$ & $425,5 \mathrm{~b}$ \\
$\mathrm{CV}(\%)$ & 6,7 & 5,5 & 2,4 & 6,7 & 1,2 & 10,0 & - \\
\hline
\end{tabular}

Médias seguidas de letras distintas na coluna são diferentes pelo teste $\mathrm{F}(\mathrm{P} \leq 0,01)$.

A proporção das membranas da casca em relação à sua espessura total foi significativamente maior $(\mathrm{P} \leq 0,05)$ em matrizes novas (Tab. 6). Este resultado assemelha-se aos de Britton (1977) e Kemps et al. (2006), que obtiveram declínio da espessura dessas membranas com o avanço da idade da matriz. Örberg (1990), Solomon (1991) e Kemps et al. (2006) demonstraram que as membranas da casca exercem influência sobre sua resistência, por servirem como reforço à porção calcificada, o que parece ter sido evidenciado neste experimento, pois os dados de resistência (Tab. 2) foram maiores em matrizes novas $(\mathrm{P} \leq 0,001)$, mesmo que as proporções das camadas vertical e paliçada tenham sido menores $(\mathrm{P} \leq 0,05)$ nestas idades. Estes dados sugerem um papel relevante das membranas da casca sobre a resistência, comparados às proporções das camadas calcificadas.

Tabela 6. Percentual das camadas da casca do ovo em relação à espessura total da casca, de acordo com a idade da matriz

\begin{tabular}{cccccc}
\hline $\begin{array}{c}\text { Idade } \\
(\text { sem })\end{array}$ & Cutícula & Camada vertical & Camada paliçada & $\begin{array}{c}\text { Camada } \\
\text { Mamilar }\end{array}$ & Membranas \\
\hline 33 & $0,65 \mathrm{a}$ & $2,66 \mathrm{~b}$ & $54,85 \mathrm{~b}$ & $25,12 \mathrm{a}$ & $16,72 \mathrm{a}$ \\
63 & $0,65 \mathrm{a}$ & $4,24 \mathrm{a}$ & $56,97 \mathrm{a}$ & $25,93 \mathrm{a}$ & $12,21 \mathrm{~b}$ \\
$\mathrm{CV}(\%)$ & 5,4 & 6,1 & 2,5 & 6,7 & 9,0 \\
\hline
\end{tabular}

Médias seguidas de letras distintas na coluna são diferentes pelo teste $\mathrm{F}(\mathrm{P} \leq 0,05)$.

A espessura da camada mamilar foi proporcionalmente semelhante $(\mathrm{P}>0,05)$ entre os ovos das duas idades de matrizes, o que demonstra que a proporção desta camada mantém-se independente da espessura da casca ou da idade da matriz. Esses dados são semelhantes aos encontrados por Ruiz e Lunam (2000), porém esses autores também encontraram proporção semelhante para a camada em paliçada, o que não foi constatado neste experimento.

Em relação à cutícula, ovos de matrizes com diferentes idades tiveram a mesma capacidade de depositar esta camada, pois, proporcionalmente, os resultados entre as idades foram semelhantes $(\mathrm{P}>0,05)$, sendo estes resultados também semelhantes aos encontrados por Ruiz e Lunam (2000).

\section{CONCLUSÕES}

A qualidade dos ovos de matrizes velhas é menor que a de ovos das matrizes novas. As membranas da casca nos ovos de matrizes novas são mais espessas e desempenham papel relevante em sua estrutura, tornando necessária ênfase a esta camada nos estudos futuros sobre qualidade da casca dos ovos incubáveis e suas relações entre idade da matriz e rendimento de incubação.

\section{AGRADECIMENTOS}

Ao INCT-PECUÁRIA, CNPq, FAPEMIG, Granja Pif-Paf, Cemel, Faculdade de Farmácia e Escola de Engenharia da UFMG. 


\section{REFERÊNCIAS}

BARBOSA, V.M. Efeitos da umidade relativa do ar na incubadora e da idade da matriz leve sobre o rendimento da incubação e desempenho da progênie. 2005. 51f. Dissertação (Mestrado em Zootecnia), Escola de Veterinária, Universidade Federal de Minas Gerais, Belo Horizonte.

BAXTER-JONES, C. Egg hygiene: Microbial contamination, significance and control. In: Tullett, S.G. (Ed). Avian Incubation. London: Butterworth-Heinemann, 1991. p.269-276.

BRAKE, J.T. Optimización del almacenaje de huevos fértiles. Avic. Prof., v.14, p.26-31, 1996.

BRITTON, W.M. Shell membranes of eggs differing in shell quality from young and old hens. Poult. Sci., v.56, p.647-653, 1977.

EMARA, O.K.A. Use of scanning electron microscopy techniques for predicting variations in eggshell quality of chickens. 2008. 252f. Dissertação (Mestrado), Department of Poultry Production, Faculty of Agriculture, Ain Shams, Alabassya.

HAMILTON, R.M.G. Methods and factors that affect the measurement of egg shell quality. Poult. Sci., v.61, p.2022-2039, 1982.

HUNTON, P. Understanding the architeture of the egg shell. W. Poult. Sci. J., v.51, p.141-147, 1995.

HUNTON, P. Research on eggshell structure and quality: An historical overview. Braz. J. Poult. Sci., v.7, p.67-71, 2005.

KEMPS, B.J.; GOVAERTS, T.; DE KETELAERE, B. The influence of line and laying period on the relationship between different eggshell and membrane strength parameters. Poult. Sci., v.85, p.1309-1317, 2006.

LA SCALA JR., N. Aspectos físicos da incubação. In: GONZALES, E.; MACARI, M. (2.Ed) Manejo da Incubação. Campinas: FACTA, 2003. p.97-124.

LUQUETTI, B.C.; GONZALEZ, E.; BRUNO, L.D.G. et al. Egg traits and physiological neonatal chick parameters from broiler breeder at different ages. Braz. J. Poult. Sci., v.6, p.13-17, 2004.
McDANIEL, G.R.; ROLAND, D.A.; COLEMAN, M.A. The effect of egg shell quality on hatchability and embryonic mortality. Poult. Sci., v.58, p.10-13, 1979.

McLOUGHLIN, L; GOUS, R.M. Efecto del tamaño del huevo en el crecimiento pre y post natal de pollitos de engorde. Avic. Prof., v.18, p.24-29, 2000.

NYS, Y.; HINCKE, M.T.; ARIAS, J.L. et al. Avian eggshell mineralization. Poult. Avian Biol. Rev., v.10, p.143-166, 1999.

ORBAN, J.I.; ROLAND SR., D.A. Correlation of eggshell quality with tibia status and other production parameters in commercial leghorns at ovoposition and 10-hour postoviposition. Poult. Sci., v.69, p.2068-2073, 1990.

ÖRBERG, J. Relationship between the shell membrane-shell bond and shell deformation in hens' eggs. Brit. Poult. Sci., v.31, p.249-254, 1990.

PARSONS, A.H. Structure of the eggshell. Poult. Sci., v.61, p.2013-2021, 1982.

PEEBLES, E.D.; BRAKE, J. Relationship of eggshell porosity to stage of embryonic development in broiler breeders. Poult. Sci., v.64, p.2388-2391, 1985.

PEEBLES, E.D.; ZUMWALT, C.D.; DOYLE, S.M. et al. Effects of breeder age and dietary fat source and level on broiler hatching egg characteristics. Poult. Sci., v.79, p.698-704, 2000.

RAHN, H. Changes in shell conductance, pores, and physical dimensions of egg and shell during the first breeding cycle of turkey hens. Poult. Sci., v.60, p.2536-2541, 1981.

ROCHA, J.S.R.; LARA, L.J.C.; BAIÃO, N.C. $e t$ al. Efeito da classificação dos ovos sobre o rendimento de incubação e os pesos do pinto e do saco vitelino. Arq. Bras. Med. Vet. Zoot., v.60, p.979-986, 2008.

RODRIGUEZ-NAVARRO, A.; KALIN, O.; NYS, Y. et al. Influence of the microstructure on the shell strength of eggs laid by hens of different ages. British Poult. Sci., v.43, p.395403, 2002. 
ROMANOFF, A.L.; ROMANOFF, A.J. (Ed). The Avian Egg. New York: John Wiley and Sons, 1949. 918p.

RUIZ, J.; LUNAM, C.A. Ultrastructural analysis of the eggshell: contribution of the individual calcified layers and the cuticle to hatchability and egg viability in broiler breeders. Brit. Poult. Sci., v.41, p.584-592, 2000.

SAMPAIO, I.B.M. (2.Ed). Estatística aplicada à experimentação animal. Belo Horizonte: FEPMVZ, 2002. 265 p.

SISTEMA de análises estatísticas e genéticas. Versão 9.1. Viçosa: UFV, 2005.

SOLOMON, S.E. (Ed). Egg e Egg Shell Quality. London: Wolfe Publishing, 1991. 149p.

STANISHEVSKAYA, O.I. Role of various zones of eggshell in gas exchange of chicken embryo. In: EUROPEAN POULTRY
CONFERENCE, 12., 2006, Verona. Anais...Verona: WPSA, 2006. p.141.(Resumo).

TAZAWA, H.; WHITTOW, G.C. Incubation physiology. In: WHITTOW, G.C. (Ed). Sturkie's Avian Physiology. London: Wolfe Publishing, 2000. p.617-634.

TULLETT, S.G.; DEEMING, D.C. The relationship between eggshell porosity and oxigen consumption of the embryo in domestic fowl. Comp. Bio. Physiol., v.72, p.529-533, 1982.

VIEIRA, S.L.; MORAN JR., E.T. Eggs and chicks from broiler breeders of extremely different age. J. Appl. Poult. Res., v.7, p.372376, 1998. 